\title{
Report on the Managing Impacts of Deep-seA reSource exploitation (MIDAS) workshop on environmental management of deep-sea mining
}

\author{
Jennifer M Durden‡, David SM Billett ${ }^{\ddagger}$, Alastair Brown§, Andrew C Dalel, Laura Gouldingף, Sabine \\ Gollner", Kevin Murphy", Ellen Pape ${ }^{\text {a }}$, Autun Purser", Jean-Francois Rolin”, Austin J Smith^, Ian \\ Stewart, Phillip J Turner^, Tom de Wachter', Philip PE Weaver ${ }^{2}$, Cindy L van Dover^, Philomene \\ Verlaan`, Daniel OB Jones ${ }^{\ddagger}$ \\ ‡ National Oceanography Centre, Southampton, United Kingdom \\ § Ocean and Earth Science, University of Southampton, National Oceanography Centre, Southampton, United Kingdom \\ I Scottish Association for Marine Science, Scottish Marine Institute, Oban, United Kingdom \\ II Environmental Resources Management Limited, Oxford, United Kingdom \\ \# Senkenberg am Meer, German Centre for Marine Biodiversity Research, Wilhelmshaven, Germany \\ a Marine Biology Research Group, Ghent University, Ghent, Belgium \\ «Alfred Wegener Institute, Bremerhaven, Germany \\ "Ifremer, Plouzane, France \\ ^Duke University Marine Lab, Nicholas School of the Environment, Duke University, Beaufort, United States of America \\ "Fugro EMU Limited, Edinburgh, United Kingdom \\ Global Sea Mineral Resources NV, Zwijndrecht, Belgium \\ Seascape Consultants Limited, Romsey, United Kingdom \\ ` University of Hawai'i at Manoa, Honolulu, United States of America
}

Corresponding author: Jennifer M Durden (jennifer.durden@noc.soton.ac.uk)

Received: 25 Aug 2016 | Published: 07 Sep 2016

Citation: Durden J, Billett D, Brown A, Dale A, Goulding L, Gollner S, Murphy K, Pape E, Purser A, Rolin J, Smith A, Stewart I, Turner P, de Wachter T, Weaver P, van Dover C, Verlaan P, Jones D (2016) Report on the Managing Impacts of Deep-seA reSource exploitation (MIDAS) workshop on environmental management of deep-sea mining. Research Ideas and Outcomes 2: e10292. doi: 10.3897/rio.2.e10292

\begin{abstract}
Background

Recent years have seen an upsurge in interest in mining in the world's deep oceans, in areas beyond national jurisdiction. Such mining activity has the potential to cause environmental impacts over large areas. As contractors plan to move from exploration to
\end{abstract}


exploitation activities it is important to develop guidance to ensure that these activities are managed in a way that is as environmentally sustainable as possible.

\section{New information}

This paper presents the outcomes of an international workshop on environmental management for deep-sea mining (DSM). The workshop presented protocols for environmental management, which were tested using a comprehensive polymetallic nodule mining scenario developed with industry input. The workshop started by presenting a framework for environmental management of deep-sea mining, which introduced the use of a conceptual model as a method of storing and updating environmental data and the synthesis of that data throughout the project. The second session focused on the incorporation of the precautionary approach and adaptive management into the framework. Two discussion sessions were held to discuss Environmental Impact Assessment (EIA) in the context of the scenario: the first considered scoping and screening EIAs, while the second reviewed the full EIA assessment. Subsequent discussion focussed on environmental management at the regional scale. This concentrated on the Regional Environmental Assessment (REA) - including its purpose, the scale and scope, and practicalities - and the assignment of protected areas as an approach for regional environmental management. Use of a scenario as a basis for the evaluation of protocols on the environmental management of DSM was novel amongst workshops, facilitating focussed discussion despite the high uncertainty surrounding DSM activities. The results of these discussions are summarised in the paper.

\section{Keywords}

Environmental management, deep-sea mining, environmental impact assessment, marine policy, environmental management plan, MIDAS project, sustainable management, European Union Framework 7, deep-sea biology

\section{Date and place}

The workshop was held at the National Oceanography Centre, European Way, Southampton, on 28-30 June 2016.

\section{List of participants}

See author list. 


\section{Introduction}

Managing Impacts of Deep-seA reSource exploitation (MIDAS; www.eu-midas.net) is a European Union-funded project under the Seventh Framework Programme. It is a collaborative project involving scientists, industry, legal experts, non-governmental organisations, and small and medium-sized enterprises. Its aim is to address fundamental environmental issues associated with deep-sea mineral resource exploitation (deep-sea mining or DSM), both in nature and scale. The project addresses the paucity of biological and ecological information available about the habitats and communities that may be impacted, and the application of existing information to guide best practice and regulations.

Recent years have seen an upsurge in the interest in mining in the world's deep oceans, in areas beyond national jurisdiction. This mining activity has the potential to cause environmental impacts through the actions of mining tools and associated impacts, such as sediment plumes. These environmental impacts have the potential to extend over very large areas, with direct impacts over hundreds of square kilometres per year (Oebius et al. 2001) and the impacts of sediment plumes likely significantly extending this area (Smith et al. 2008). As contractors plan to move from exploration to exploitation activities it is important to develop guidance to ensure that these activities are managed in a way that is as environmentally sustainable as possible.

Critical components of the MIDAS project are documenting the best environmental practice for the exploitation of deep-sea minerals, and developing a set of protocols to be used by industry to improve the environmental sustainability of their operations. These protocols will comprise the overarching environmental management of the project, including impact assessment and monitoring, at both project and regional scales. It is important that the protocols are evidence-based, in addition to being practical to implement.

\section{Aims of the workshop}

The aims of the workshop were to:

- test the application of environmental management protocols using a deep-sea mining scenario;

- document gaps, inconsistencies and other areas for improvement in the draft protocols; and

- demonstrate to other work packages how their scientific information may be applied.

To solicit opinions from experts of diverse backgrounds, the workshop involved scientists from each of the work packages of the MIDAS project, legal experts, and industry representatives. Here we summarise the results of the discussions at the workshop.

The protocols to be assessed fell into three broad categories, which were each allotted one day of workshop time: (1) a framework for environmental management, (2) DSM project- 
scale or site-specific Environmental Impact Assessment (EIA) and Environmental Management Plans (EMP), and (3) regional-scale environmental management of DSM. The protocols included:

- a draft framework for the complete environmental management for a DSM project, encompassing the phases and components therein, indicating responsibilities and tasks for both the contractor and regulator;

- draft guidance on environmental management areas within a DSM claim area, and the definition of further areas necessary for robust environmental management;

- $\quad$ the guidance for EIA and EMP provided by the International Seabed Authority (ISA; International Seabed Authority 2011); and,

- a description of considerations for regional-scale environmental assessment (REA), including options for the assessment of protected areas (known as Areas of Potential Environmental Interest; APEls).

The protocols were tested using a comprehensive nodule mining scenario developed as part of MIDAS project work package 7 , in collaboration with industry partners. The scenario was focused on the near-seabed processes of nodule mining at a claim area in the ClarionClipperton Zone (CCZ) in the Pacific Ocean. Data on a mining vehicle and on-site mineral processing plans were provided by IHC, while environmental data were provided by BGR or collected as part of a MIDAS project research cruise to the eastern CCZ in 2014 (RRS James Cook 120). Representative sediment plume models were created by Andrew Dale (SAMS). The use of a specific scenario as a basis for the evaluation of protocols was novel amongst workshops on the environmental management of DSM.

\section{Key outcomes and discussions}

\section{Methods of capturing discussion outputs}

The protocols were assessed over eight discussion sessions. The workshop was highly structured, with discussion questions assigned to small discussion groups (3-5 attendees each). In each session, attendees were randomly assigned to one of four groups, with one attendee per group assigned to be the discussion manager, and one to be the reporter. The discussion manager facilitated discussion in each group, and was specifically tasked with ensuring that all assigned questions were addressed, and obtaining input from all group members. The reporter captured qualitative responses for each group, recorded these outputs into a standard document template, and submitted to the workshop organisers at the close of each day. At the end of each discussion session, the reporter also provided a short verbal summary of the group's outputs to the complete workshop group.

Attendees were surveyed at four points throughout the workshop: at the beginning of the workshop, and after each group of sessions. Some survey questions were designed to provide further results at the conclusion of a workshop session, whereas other questions were asked periodically to provide insight into the development of attitudes towards the environmental management of deep-sea mining. Surveying was conducted using electronic 
polling with hand-held voting consoles through a PowerPoint interface (TurningPoint 5), facilitating instant feedback of results. Voting by attendees was not mandatory.

\section{Environmental Management Framework}

Two discussion sessions were held to evaluate the framework for the environmental management of a DSM project. The focus of the first session was on the use of a conceptual model as a method of storing and updating the environmental data and the synthesis of that data throughout the project. The second session focused on the incorporation of the precautionary approach and adaptive management into the framework.

The following questions were posed to focus the discussion on the use of the conceptual model within the framework:

- Follow through the framework using the scenario. What resource/mining technology/environmental knowledge is needed or improved/increased at each stage of the process?

- Does updating a conceptual model make sense in terms of following scientific understanding of the environment? What gaps are there?

- How would the information in a conceptual model best be presented, stored and shared? Are the reporting points in the framework appropriate?

- How could the updating of a conceptual model be facilitated and enforced?

- Does the framework fit in to existing environmental management guidance and project phase requirements from the ISA? Are there inconsistencies? If so, what are they?

- Would this framework be more or less applicable to another type of deep-sea mining? If so, how?

After reviewing the framework, attendees agreed that it predominantly fit the project phases of DSM and the existing environmental management guidance published by the ISA. The framework was assessed as following the development of scientific understanding of the environment, reflecting the dynamic process of gaining knowledge during project phases, while incorporating milestones to provide some stability to the process. The mechanism to facilitate updating the conceptual model was identified as the inclusion of review phases at junctions where regulatory approval is required for the progression of contractor activities. It was highlighted that this may be obvious for junctures such as EIA preparation and approval prior to exploitation licensing, but that specific mechanisms may be required to ensure periodic review, for example during times when mining is suspended and when annual monitoring is required. Attendees also stressed that contractors should be required to establish an accredited (e.g. to ISO 14001) environmental management system to inform the early stages of the conceptual model.

Concern was expressed that the expectations for environmental management in phases prior to exploitation of polymetallic nodules have already been specified by the ISA, since many contractors are nearing the end of their licenced exploration phases. These may need to be revised to include more robust data collection, interpretation and review, as 
suggested by the conceptual model in the framework. However, this may be more easily accomplished for other types of mining, where exploitation guidance has not been established.

The main source of initial information for environmental management, the regional environmental assessment (REA), was evaluated as needing substantial development. Currently, no formal REA exists, although some regional-scale data are available (e.g. productivity gradients and nodule density differences used in the design of the APEIs; Lodge et al. 2014; Wedding et al. 2013). Attendees identified that information is needed from the REA to inform the environmental management planning for a project. This should include all previously published scientific studies from the region on the habitat and environment, including geographic, physical, chemical, biological and geological data and interpretations thereof, and descriptions of the scale and the nature of natural spatial and temporal variation (including episodic and periodic events).

Attendees were more certain of some portions of the framework (corresponding to phases of a DSM project) than others. While the ISA has considered EIA and EMP, some environmental data has been collected, and contractors are developing extraction techniques, the technical and practical aspects of rehabilitation are almost completely unknown, making this aspect particularly difficult to plan. Attendees were not in agreement on the necessity of long-term post-mining monitoring, including the time scale. It was identified that the natural recovery of nodules would involve long time-scales, such that monitoring on this temporal scale may not be economically desirable for contractors. One suggestion was that an intermediate monitoring time scale could be equivalent in length to the exploitation duration, following cessation of mining.

Two additional phases were also thought to be potentially important: screening and test mining. Screening is part of the initial desk study phase, and includes a review of whether mining is feasible and economically attractive. Attendees suggested that information from the REA could help to estimate the environmental liability at this stage, and that communication with the regulator about environmental management at this early stage was desirable. In addition, information including in this screening phase should be included in the EIA. It has been assumed (by the ISA and others) that test mining will be conducted prior to full-scale exploitation activities, so some attendees suggested that this phase should be included as a separate stage in the framework. Others thought that it could be part of the described work, potentially as a phase of the exploitation activities set out in the mining plans. A concern was raised related to the practical logistics and contractors' expectations related to the suspension of test mining for a time sufficient to review environmental data on impacts, review and revise the EIA, and complete a regulatory review prior to embarking on full scale mining.

Although the ISA has published a list of useful parameters to sample/monitor (International Seabed Authority 2011), further details on these parameters is needed, including minimum sampling requirements, methods for sampling and analysis (including spatial sampling design and temporal frequency), and thresholds for action. In essence, the regulator should set expectations for how much research is required. Such detail would improve the quality 
of the data collected in the baseline study, EIA preparation and monitoring, and simplify updating and interpreting the conceptual model, in addition to facilitating data exchange between contractors and with the regulator. Attendees thought that conceptual model updates should include review of information from other contractors' experiences, not just a single contractor's own data.

Workshop attendees agreed that the information in the conceptual model must be made publically available, and stored in a standardised format that can be accessed online. In addition to making available the data behind the conceptual model, levels of certainty/ uncertainty should be defined, descriptions of interpretations, and reasoning behind decisions made should be included in order to increase transparency and facilitate future iterations.

The framework was seen to be equally applicable to other types of DSM. However, the smaller spatial and particularly temporal scales of seabed massive sulphides (SMS) and crust mining may require different timescales for monitoring, updating and reviewing the conceptual model. The areal extent of projects defined will also vary between mining types.

The following questions were used to guide the discussion on incorporation of the precautionary approach and adaptive management into the scenario:

- Review the framework in the context of the scenario. At what points in the mining/ environmental management process should the precautionary approach be included? Are these captured in the framework?

- At what points in the process could adaptive management be used? What should or could it be used for at each? Are these possibilities captured in the framework?

- Does the framework capture the monitoring and review necessary for adaptive management at reasonable points in the project process?

- How could the application of the precautionary approach and adaptive management complement one another in the framework? How might they oppose?

- What reviews or controls would need to be in place for successful adaptive management? Are these points identified in the framework?

- Have opportunities for incorporating adaptive management and the precautionary approach been missed? If so, what are they and where?

Attendees agreed that the precautionary approach should be considered at all phases of a DSM project, and thus at all stages of the framework. The precautionary approach applies to all areas where uncertainty exists, whether related to environmental understanding (as this is developed), technical aspects of the exploration methodology (approaches, methods and tools), the environmental management process (as required by the ISA, or as part of a contractor's environmental management system), or the regulatory requirements (including changing regulations or thresholds as more is known). Uncertainty in these areas exists at both the project-specific and regional scales. Again, attendees suggested that these uncertainties should be recorded in the updated conceptual model, along with how the precautionary approach was incorporated into decision making at each stage. It was 
concluded that the framework provided provision for the implementation of the precautionary approach.

The precautionary approach is most effective when considered in advance. For example, if it is to be applied to the monitoring of environmental impacts of mining activities (with certain parameters to be measured certain locations), then it is important to consider these impacts and parameters when assessing the environmental baseline to ensure that sufficient data is collected for comparison (e.g. at the same locations, for the same parameters, using standardised methods). It was noted that in following a precautionary approach, more phases of testing (of mining and environmental sampling/monitoring techniques, methods and tools) may be required, along with more frequent reviews (at short time intervals) of each phase by contractors and regulators (including monitoring/ sampling and auditing), in situations with less certainty than in those where more information is available and more certainty is involved. Again, it was noted that decommissioning and rehabilitation is a phase with little knowledge and high uncertainty.

At the centre of the discussion was the practical definition of 'precaution'. While it was agreed that the regulator must set the standard for precaution in each area, a reasonable approach to precaution was not agreed upon. Some suggested that the threshold for precaution should be zero harm, others that impacts should not exceed natural variability in time and space, while some thought that an 'acceptable impact' could include impacts to a maximum area and/or within a timeframe where the wider ecosystem is still able to function and recover. The threshold must be considered against a worst realistic impact case from the mining plan. Regardless of the initial required level of precaution, the level of precaution applied should be reassessed as projects progress to ensure that allowable risks become smaller as more information is known. The regulator must consider precaution at both the regional and project-scale when evaluating contractors' plans and work. Attendees also agreed that the regulator must set out consequences for not adhering to the approach and the threshold, including not granting or withdrawing approval for mining activities, and the imposition of financial penalties.

Attendees also agreed that the framework included provision for adaptive management in a DSM project. Several points made were common to those on the precautionary approach: the need to identify and document uncertainties within the conceptual model and how adaptive management may be used to address them; the increased importance of the use of adaptive management in phases with less certainty; and the need for adaptive management to incorporate multiple adaptations (to technical aspects of mining, environmental understanding at the project and regional scales, technologies and regulations). The framework could be modified to reflect the required engagement of stakeholders, including the scientific community, in adaptive management. A major concern with the inclusion of adaptive management in the framework was the definition of timescales, such as the time required to collect sufficient information to inform the adaptation sought, and the time for which approval for an adaptation should be valid.

Testing, particularly test mining but also test monitoring, was revisited in the discussion, as it would provide information useful to the full-scale EIA and EMP. The need for a distinct 
test mining phase in the framework was discussed, with the possibility of a separate EIA requirement for test mining, in contrast to allowing the test mining process to be captured through an adaptive management loop. It was pointed out that the time lag between test mining and full-scale mining will likely be insufficient to capture robust information about anything other than immediate effects and short-term recovery, particularly in the case of nodule mining. Over a longer timeframe, the test mining and commercial mining as well as attempted mitigation actions will build up the evidence base for future mining activities, this information is an important part of the conceptual model.

The use of adaptive management loops involving monitoring and auditing during mining activities (both test mining and full-scale mining) was discussed. This feedback loop should be explicitly required during exploitation, including the updating of the conceptual model and adjustment of the mining plan. It was noted that such adaptive management should be included at earlier stages, such as during exploration (e.g. auditing of the environmental management system).

Attendees noted that the precautionary approach and adaptive management can be complementary if the latter is implemented effectively, because environmental impacts that are anticipated, modelled and/or detected may be evaluated and a mitigation plan implemented. However, the use of adaptive management to facilitate experimentation may be in opposition to the precautionary approach, particularly if the experimentation is extreme in its nature. Such experimentation may nevertheless be necessary to establish thresholds for precaution.

Successful adaptive management is dependent on adequate legal and regulatory mechanisms and resources being in place to revisit and modify the conceptual model and plans (e.g. EIA and EMP), and regulatory approval at all phases. Attendees suggested that conditions in the regulatory approval for exploitation (and exploration) as part of licenses or contracts should include conditions of monitoring and reporting at each phase in the framework, requiring regulatory review and updating of the EMP. Consequences must be defined for breaching such conditions, including the suspension or cessation of mining activities. In addition, the regulator must define situations requiring a new EIA/EMP and those that can be accommodated through adaptive management. It was suggested that the precautionary approach should be the basis of decision making, and that the opportunity to implement adaptive management (allowing a greater scope for mining activities, and experimentation) should only be allowed by the regulator once a contractor has proven its commitment to robust environmental management (for example through successful audits, report submission, etc. as the framework progresses).

Finally, the main concern with the implementation of the framework, and environmental management of DSM generally, was the capacity of the regulator to review information and provide feedback at necessary intervals. The regulatory body must have a good technical understanding of the environmental risks, and technical and environmental requirements of mining, with sufficient resources to interact with contractors at all times. They must also ensure the accountability of contractors through reviews and auditing, tasks that require a substantial allocation of qualified professionals, with appropriate resources. Such 
regulatory resource must be available to accommodate the needs of all DSM contractors over the long-term (e.g. to include long-term monitoring in the decommissioning phase) to facilitate and ensure contractors' best environmental performance. Attendees identified that structural reform to provide a permanent competent regulator would allow responsiveness to facilitate development of dialogue between regulator and contractors and stakeholders. The current regulatory structure, expertise and resources of the ISA are insufficient to meet this need.

Overall, the framework was viewed as a useful tool to assist the ISA in its requirement to protect the environment (42\% indicated 'greatly', 50\% indicated 'moderately', $8 \%$ indicated 'somewhat', $n=12$ ). The establishment of the framework was also viewed favourably for reducing uncertainty in the environmental management process $(21 \%$ indicated 'greatly', $50 \%$ 'moderately', $29 \%$ 'somewhat', $n=14$ ). It was also seen to be a tool by which the ISA could ensure fairness and standardisation between contractors (42\% indicated 'greatly', $33 \%$ 'moderately', 17\% 'somewhat', $8 \%$ 'slightly', $n=12$ ).

\section{Definition of areas and timelines important to environmental management}

The aim of this session was twofold: to consider and evaluate environmental management of DSM in terms of space and time, with reference to the environmental management framework. The discussion groups were divided, with two groups considering each aspect. It was acknowledged that separating spatial and temporal considerations is difficult because they often interact; for example, processes may operate over timescales at different spatial scales (e.g. succession).

To consider appropriate spatial scales and defined areas for the environmental management of DSM, the following questions were posed to the discussion groups:

- $\quad$ Are the areas appropriate to match their purpose? Are any further or fewer areas needed? If so, what and for what purpose?

- Review the defined areas diagram in the context of the scenario. Where should each of the areas be located? What additional considerations are necessary? Is any further information needed about the scenario to define the areas? Are any conflicts between areas foreseen?

The discussions generally concluded that the defined areas were sound in theory for nodule mining areas, and made some common recommendations about all areas. The size and locations of defined areas should be determined based on data on spatial environmental heterogeneity and variability provided by the claim baseline study and studies of other areas in the region, and based on sediment plume modelling, with minimum sizes established by the regulator. Areas must be representative, which should be determined by all environmental metrics (e.g. nodule abundance, productivity, geomorphology, faunal density/biomass and diversity). Consideration for the numbers of replicate areas per type should be made with the aim of producing statistically robust comparisons from the resulting monitoring data, and experts in survey design should be 
consulted in the determination and review of areas. The before, after, control, impact (BACI) approach was recommended (Underwood 1992).

The concern over the potential for moving control areas (such as the APEls and preservation reference zones) was common to several discussion groups. The prevailing opinion was that control sites should be established at the beginning of the project, monitored sufficiently, and never moved. Furthermore, future phases of mining must continue these areas, preserving their original purpose. For example, preservation reference zones must be protected from sediment plumes.

Additional areas deemed important for monitoring were discussed in the workshop and recommendations were made. It was felt important that there were (1) impact reference sites for sediment plumes (locations which receive sediment plume impact but not direct mining impact), which should be situated both within and outside areas suitable for mining (two habitats); (2) preservation reference zones, which should be monitored and not impacted, in areas not suited to mining but with other features of ecological interest (e.g. seamounts).

The additional areas defined were found to be important for spatial environmental management $(90 \%$ indicated 'greatly' and $10 \%$ indicated 'moderately', $n=10)$. The possibility of sediment plumes extending outside the claim area, and possibly into other claim areas and/or APEls was identified as being important to detect and mitigate against. While monitoring of sediment plume extent outside the claim area was seen to be important, concern was expressed for the practicalities of doing so, and the regulatory mechanisms to require and facilitate such monitoring.

To consider appropriate timescales involved in environmental management of DSM, the following questions were posed to the discussion groups:

- On what timescales would mining-related processes occur in the scenario? On what timescales would project management-related processes occur in the scenario? On what timescales would environment-related processes occur in the scenario? Do any of these timescales match up between the three? Are these matches related to stages in the framework? What processes/timescales do not match? What challenges does this pose for environmental management? How might these challenges be overcome? What changes to the framework would be needed?

- How could stopping/starting of mining activity be incorporated?

- How might these defined areas and timescales differ in the context of another type of mining?

Although the scenario specified a timescale of 25 years for mining operations, the discussion identified important timescales of mining-related processes within that period. These including phased mining (such as test mining followed by full-scale exploitation, or multiple phases of full-scale mining within the 25 years), planned pauses in extraction (e.g. for technical reasons, or to assess monitoring data), and unplanned extraction downtime. 
Project management-related processes identified included collection of environmental baseline data and resource assessment (e.g. 5-15 years), and exploitation (25 years). Timelines for technical changes could be instant (e.g. change of seabed vehicle direction) or many years long (e.g. design and installation of new equipment). Environmental processes in the context of the scenario occur at different timescales, where they are known (or reasonably estimated). For example, tides (daily and spring-neap variation), eddy passage (months), currents (seasonal), organic matter input to the deep-sea (seasonal), climatic variations (inter-annual, e.g. El Niño/La Niña and climate change), softsediment faunal recovery from removal (century-scale), nodule recovery from removal ( tens of millions of years).

Participants agreed that appropriate timescales involved in the environmental management of deep-sea mining were difficult to define, because operational and project management timescales do not necessarily match timescales involved in environmental processes (where known). Currently, the framework and the enacted processes for mining approval are related to the operational and management timescale, not considering those of environmental processes. In particular, timescales of natural variability may not match timescales for baseline data collection. Furthermore, since the timescales for habitat and benthic community recovery are immense, it will be impossible to use data from monitoring such recovery at one site to feedback into good decision making for subsequent mining projects.

Despite the difficulties, participants voted that defined timelines would greatly $(69 \%)$ or moderately (31\%) improve the environmental management of DSM $(n=10)$. Several suggestions were made to accommodate environmental timescales within DSM: (1) sustained observations are necessary to provide further data on timescales of natural variation in the environment (thus constancy of monitoring location is important), (2) phased mining at different locations would allow new information gained from early phases to be used in timing later phases, (3) timescales should be considered in operational planning (e.g. avoid mining certain locations at certain times, for example to accommodate seasonal changes in currents), and (4) conditions in the mining contract should include the cessation of mining if temporal conditions change.

Finally, scales of time and space in other types of mining were identified to be different to those for nodule mining. Mining of seabed massive sulphides would happen on a much smaller spatial scale than that of nodules, and cobalt crust mining at an intermediate spatial scale, potentially leaving little space for the defined areas identified (e.g. reference zones). Thus, the size, number and nature of different areas may need to be adjusted. Recovery time was identified to occur on a shorter timescale at hydrothermal vents than for nodule environments (possibly $\sim 10$ years for vent communities, with longer times for off-vent communities). Recovery from mining of cobalt crusts was estimated to occur on an intermediate timescale (tens to hundreds of years). 


\section{Environmental Impact Assessment}

Two discussion sessions were held to discuss EIA. The first considered scoping and screening EIAs, while the second reviewed the full EIA assessment, both in the context of the scenario.

The following questions were posed to focus the discussion on scoping and screening EIAs:

- Review the scenario, and complete a scoping EIA (risk assessment). What information should be included? Identify what information is needed from the REA, from previous studies/literature, from the contractor, from the ISA, or from other sources.

- What types of activities should require a screening EIA?

- For how long should a scoping EIA be valid (minimum and maximum)? What factors should be considered when setting this timeframe?

- What regulatory mechanism(s) would be needed to facilitate communication between the regulator, contractor and stakeholders in the EIA process? How could this communication be enforced?

- What criteria should be used to evaluate and accept/reject a scoping EIA? Consider the criteria with respect to the precautionary approach, and adaptive management.

The premise of the scoping EIA is to define the scope of the project and the full EIA. The assessment is based on knowledge of the nature of the project, knowledge of the environmental setting, the regulatory and other requirements, and stakeholder views. This evidence base is lacking in the case of DSM, and thus extensive work is required before a project could proceed. Substantial professional judgement based on this evidence is required, with few qualified people currently available to provide this input.

The scoping EIA must include both the assessment of planned impacts (e.g. mining), with the degree of confidence in their anticipated impact, and risk assessment of unplanned impacts (e.g. resulting from spills or earthquakes). To conduct a robust scoping EIA, extensive information on the environment is needed, a comprehensive risk source register must be established, and a solid quantified understanding of responses to such risk sources is necessary. A primary aim is to identify headline risks and key impacts specific to the project. Much of this information is needed at the project scale, but information on the regional environmental conditions and other impacts is also required.

It was agreed that material changes to the project (even after license), in terms of mining method and plan, to the environmental baseline or to the wider evidence base should require a screening EIA. Changes to the mining approach include any that would alter the spatial or temporal scope, severity, or nature of the potential impacts, and may include timescale for mining, changes to seabed extraction methods (e.g. mechanical vs hydraulic), changes to the nodule processing (e.g. at the seabed or shipboard, use of chemicals), changes to storage and transfer systems (for nodules and chemicals), changes to discharge of return fluid (e.g. composition, flow rate, temperature and pressure, method, 
timing and location). Smaller changes to activities, such as changes to hydraulic fluid in seabed vehicles, may only require an addendum to the EIA. Other small scale activities, such as pilot mining, scientific experiments and mining vehicle prototype testing on a small seabed area (i.e. $<10,000 \mathrm{~m}^{2}$ ), may not require a mandatory EIA, but could benefit from a voluntary EIA.

The scoping EIA must be revisited regularly during the full EIA assessment. Once this is finished, the scoping EIA report should be valid for a considerable time. Attendees suggested that in the absence of changes to the planned mining activities or to the environmental assessment, then the scoping EIA should be reviewed, updated and resubmitted for approval every 5 years. This review should consider any improvements to the understanding of potential impacts as more information is collected (by the contractor or others) that may change the conclusions of the scoping EIA.

The regulator should establish the requirement for a scoping EIA, submission of a scoping EIA report, and consultation. This process should be a requirement, along with the full EIA and EMP, for regulatory approval and for the exploitation license or contract to be issued. Scoping EIA reports may also be required to secure financing from funding agencies. The required consultation must be stipulated by the regulator, but should include major stakeholders, the scientific community and the public. Consultation should be made on the scope of the EIA and the finalised scoping report.

The regulator must also define the criteria by which a scoping EIA report would be evaluated. It must be comprehensive, based on robust methodology, and be peer reviewed. The report should be accessible and be made available. Attendees suggested that the following components must be present in an acceptable scoping EIA:

- A description of compliance with existing law

- A comprehensive review of available information from scientific literature, the regulator (e.g. REA and regulations), existing environmental information on the claim

- Realistic mining plan scenarios using real data

- $\quad$ Evidence of use of best available technologies and best practices

- Evidence of integration of the precautionary approach

- Levels of certainty/uncertainty must be described

- Identification of points where adaptive management are to be applied, and a proposal of the adaptation

- Comprehensive plans for acquisition of remaining/further data

- Comprehensive plans for assessing relevant risks

- Results of stakeholder consultation, and a list of those consulted

Finally, attendees generally agreed that a regulator-reviewed scoping EIA should be a necessary phase of environmental management of DSM (93\% indicated 'greatly', 7\% 'slightly', n=14). 
The following questions were used to guide the discussions on full EIA assessments:

- Review the scenario, and complete the EIA checksheet (in International Seabed Authority 2011). Consider the EIA in terms of the spatial management areas defined. How could the conceptual model be used to inform the full EIA?

- If the full EIA is accepted, what conditions should be considered or mandated in an extraction license? For how long should the full EIA be valid?

- What mechanism(s) could be used to ensure that adaptive management (including experiments) could be implemented into the mine plan and EIA? How could adaptive management measures be monitored, reported on and enforced?

- What type of change to mining plans would require a revision/updating of the full EIA? On what timescales might these occur?

Attendees found that the categories of information required in the EIA checksheet were reasonably comprehensive, and based on EIA processes from other industries. However, the level of detail required was not clear, including the methods and standards used to acquire the data. They identified three major components missing from the checksheet:

- A description of the development of the EIA, including how it has been shaped, and by what factors, and also what approach and methodology were used in developing the EIA. This should in the Introduction.

- The checksheet needs to include the minimum standards for data acquisition, including what sources must be consulted. In addition, in the description of the environment, the minimum standards for quantification must be listed (e.g. how many samples collected where and when, collected with which method, analysed using which method, etc.).

- A scientifically-sound synthesis of the information included must be required. This may be the most important portion of the EIA, in essence 'what does it all mean?' It must include a combined consideration of all information, including interrelationships, cumulative impacts and knock-on effects, and their relative importance to the overall environment. Combinations should be of environmental conditions (e.g. monitoring data of physical, chemical, biological, geological parameters), and also identified risks. Each risk combination should be evaluated both ways (e.g. How do natural hazards and mining impact biological communities when combined? Does the natural hazard exacerbate the mining impact? Does the mining activity cause/increase the natural hazard effect?). Modelling could be used here to assess and illustrate the synthesis.

Further suggestions of missing information were made at a more detailed level. The introduction (purpose and justification) should include an environmental aspect and goals, along with an assessment of the viability of the project. The description of the proposed development should include definition in both space and time. The former should include reference to and description of all spatial management areas, while the latter should include not only the project timeline (e.g. phases of mining activity), but also timelines and frequency of monitoring, and the anticipated timeline of impact (e.g. duration of sediment plumes at a particular extent, target time for curtailing such plumes, and timelines for 
decommissioning and rehabilitation). Computer modelling is necessary to assess the environmental risks in space and in time, and in considering the spatial management areas with these timelines.

The conceptual model was considered by attendees to be key to storing all of the information necessary for the EIA, and for all other phases of the project. As it contains all historical information on the environment and mining activities it can and should feed into all sections of the EIA. It was considered to be the 'most important tool for contractors' to achieve good environmental management.

Attendees agreed that the development of the full EIA should be an iterative process requiring (regular) interaction between the contractor and the regulator from an early stage, so that mitigation is built into the project design. Mitigation decisions should be documented, including a description of the mitigation options considered for each risk, rationale for the chosen mitigation over other options, its practical implementation, its desired effect, and the plan to monitor its effect. Such a process would benefit both the contractor and the environment, and would require substantial resource allocation by the regulator. They also suggested that the EIA should be prepared by a qualified, independent party.

Attendees also agreed that the development of a full EIA would be eased and improved by having an existing REA. The REA would provide a regional overview of environmental conditions (including historical information), combined effects of other activities, and prioritise potential impacts. The approach for major topics, such as climate change, should be identified from the REA, with adaptation and mitigation addressed in both the EIA and REA.

The criteria and mechanism for the evaluation of the full EIA, and enforcement of the mitigation measures in it, must be set out by the regulator in advance of EIA preparation. In evaluating the EIA, the results of consultation with stakeholders on the baseline environmental study, and draft EIA must be considered. The EIA should also be peerreviewed by qualified/competent people, with the results considered in the regulatory evaluation. Evaluation criteria should also include a review of the previous environmental performance of the contractor, with extraction licenses/contracts withheld for contractors with poor performance.

Attendees agreed that some mandatory conditions must be placed on an extraction license or contract issued after the EIA has been accepted. These would include: (1) the adherence of the contractor to the mining plan and mitigation measures; (2) monitoring and reporting of these, as laid out in the EIA; (3) being subject to independent auditing to confirm such adherence; and (4) mandatory periodic reviews of the EIA and monitoring results. These are standard conditions to continued operation in allied industries, specifically including standardised monitoring and reporting requirements, information dissemination and audit parameters (type, frequency, reporting on findings, and potential consequences). In addition, conditions related to the implementation of adaptive management must be included (e.g. triggers, decision-making processes, and timelines for 
data collection, review, feedback, and alteration of the EIA). Other conditions should include the protection of the preservation reference zones and APEls, and vulnerable species. Finally, the conditions must include triggers to stop or pause mining, due to breaches of the above, or external factors (e.g. impact from adjacent claim, alteration to the environment from a natural hazard, etc.).

Conditions considered in an extraction license could also include a set of performance standards, imposed by the regulator, which could become more stringent through time (i.e. adaptive management of performance standards). An example of this can be found in the air emissions limits set by the EU. These performance standards should be quantifiable, enabling their reporting. Performance against the standards could be monitored by the regulator using in-situ measuring devices and remote sensing technology. Not complying with these standards should result in the contractors having to either pay a fine or lose their environmental bond to the regulator, or another constraint on operation.

The length of validity of an EIA was debated, with suggestions to review the full EIA at intervals of 5,20 or 25 years (unless otherwise initiated as described below), in addition to the review following the annual monitoring reporting. The range of review frequency was suggested on the basis of time frames to collect sufficient environmental data or to encourage the development of technology (5 years), or for financial commitments (20-25 years). The regulator must stipulate which activities or changes (e.g. to the environmental understanding, or events caused by nature or contractors) require an EIA amendment, or an adaptive management procedure, or a full new EIA (see scoping, above).

Upon polling, attendees largely considered the ISA's EIA requirement (International Seabed Authority, 2011$)$ only to be 'somewhat' complete $(75 \%)$, while a few attendees consider it to be 'greatly' (8\%), 'moderately' (8\%), and 'slightly' $(8 \%, n=12)$. They also differed on the priority to improve EIA: an equal portion of attendees prioritised the definition of criteria for evaluation of the EIA (43\%) and establishing an REA (43\%), while a few would emphasize requiring regulator input at the scoping EIA (7\%) or developing guidance on sampling methods for individual parameters $(7 \%)$, and none prioritised the definition of areas for environmental management (described above), or increasing knowledge of the mining plan $(n=14)$.

\section{Environmental Management Plan}

The Environmental Management Plan (EMP, also known elsewhere as the Environmental Management and Monitoring Plan) is created using the outcomes of the EIA, so the discussion session followed those on EIA. The discussion was focussed around these questions:

- $\quad$ Try to develop an EMP in the context of the scenario and the EIA, identifying what information is needed.

- What criteria should be used to evaluate the EMP? What timeline should be involved? For how long should an EMP be valid? 
- How should the conceptual model of the claim be updated during extraction activities? At what time intervals?

- How should the mitigation hierarchy be implemented into the EMP?

- What mechanism(s) is/are necessary for communication between the regulator and contractor, and stakeholders during the development and evaluation of the EMP?

- If the EMP is accepted, what conditions should be considered or mandated in an extraction license?

The EMP is prepared using the information from the baseline study and the approved full EIA. The current EMP requirement (International Seabed Authority 2011) was found to be only 'somewhat' $(67 \%)$, or 'slightly' complete $(33 \%, n=12)$ by workshop attendees. They suggested that the EMP must include

- details of the practical implementation of all mitigation measures identified in the EIA, to ensure that all thresholds are met;

- details of the monitoring plan (including spatial design and frequency, methods, parameters, analyses, intended comparisons to previous and concurrent data and thresholds);

- details of the contractors' environmental management system, and how it will be used to implement the mitigation measures and monitoring;

- $\quad$ roles and responsibilities of all parties;

- descriptions of anticipated and completed adaptive management; and,

- results of monitoring (as they become available) and adjustments to the EMP based on the updated conceptual model, including rationale and details of regulatory approval.

Communication between the regulator, contractor and operational personnel of the contractor is vital to the successful implementation of the EMP. This should extend to subcontractor and independent auditors. Methods of communication must be established and recorded in the EMP and the contractor's environmental management system.

Attendees agreed that the regulator must establish the criteria by which an EMP is to be evaluated, with minimum requirements. This must include all items listed above. It must prioritise the mitigation measures for the major effects listed in the EIA, and result in the compliance with the environmental standards set by the regulator. Similarly, the regulator must define their methods for evaluating adherence to these requirements, including auditing. Contractors' EMP and (annual) monitoring reports, and reports evaluating the contractors' performance of the EMP should be made publically available.

As the EMP is produced upon completion of the EIA, it must be reviewed whenever the EIA is amended. It should also be reviewed by the regulator (and amended as necessary) following the completion of monitoring reports, any adaptive management activities, or the update of the conceptual model. The EMP should be valid for the same period as the EIA, with the condition of these reviews. 
Upon regulatory approval of an EMP, several key conditions must be included in the extraction license or contract:

- all environmental objectives are met;

- $\quad$ all processes and procedures in the EMP are followed;

- monitoring is completed as stipulated in the EMP, and reports are submitted to the regulator at least annually;

- the annual approval of the EMP following review (if approval is warranted);

- $\quad$ submission to audit by the regulator;

- a mechanism to inform the regulator of any contravention or unplanned event; and,

- acceptance of the regulator's imposed consequences, including possible suspension of mining license/contract, when applicable.

\section{Regional Environmental Management}

The discussion on environmental management at the regional scale was divided into two sessions. The first concentrated on the REA, including the scale and scope, its purpose, and practicalities. The second concentrated on the APEls, as protected areas, in the context of regional environmental management.

The following questions were used to direct the first discussion session:

- Review the REA description in the context of the scenario, and its location in the CCZ. In reference to the scenario, what information should be included in the REA? Over what spatial and temporal scales would this need to be collected and assessed?

- How might the REA be used/developed to assess large-scale change? Would this require any changes to the content or timeline for updates?

- How might the REA be used/developed to compare impacts between regions? Would this require any changes to the content or timeline for updates?

- $\quad$ Are the areas proposed for the 'regions' appropriate? Why or why not? What factors should be used to determine the 'region'?

- What minimum information is required in an REA before contractors' extraction activities are begun?

- How might REA be used to incorporate active adaptive management / experimentation / development in deep-sea mining? Would this require any changes to the content or timeline for updates?

- How would the REA differ for different mining types?

The REA should facilitate overall environmental management, and set overall environmental objectives for the whole CCZ; that is, it should be an active document, rather than a passive one as suggested by the Operational and Management objectives for the CCZ in ISBA/17/LTC/7 (Sections VIA and VIIA). These environmental objectives should include overall aims for the preservation of biodiversity, for example. It should aim to identify the regional resource distribution and environmentally sensitive areas, and include a regional environmental model. Ideally the mining exploration and exploitation claim areas 
and areas for preservation should be allocated based on this regional assessment. The REA should identify potential conflicts with environmental objectives and aim to reduce cumulative effects, while being improved by the iterative integration of all contractors' environmental data. It should proactively determine whether contractors are gathering data at a scale appropriate to addressing environmental management issues, and provide guidance to the development, assessment and approval of EIAs and to ensure adequate data collection (not only at a claim scale). Mechanisms for joint management of multiple contractors, and different business sectors (e.g. DSM and fisheries) are required.

Several options for the area of the 'region' described by the REA were discussed, including the size of a claim (or maximum extent of multiple claim parts for those that are discontinuous), the size of multiple claims and at least one APEI, dividing the CCZ into six parts (as done when the APEls were designated), or the CCZ as a whole. The latter was thought by some to be too big for effective management. Some attendees suggested that a nested approach would be the most appropriate. Others suggested an ecological approach rather than a geographic one, where the region was determined by ecological, connectivity and management objectives. In any case, the outer boundaries of the management area need to be established.

At the large scale, the REA would be used to determine which activities pose the greatest environmental risks, to assess and manage the interactions of DSM with climate change, fisheries, large-scale natural hazards (e.g. weather patterns such as El Niño). Concepts from landscape ecology, such as the maintenance of regional scale ecology, sources and sinks, and biological corridors, could be implemented in the regional management. At the intermediate scale, the REA could be used to coordinate contractors' activities and studies across contractors' claim areas, and to identify the potential for conflicts (e.g. plumes crossing claim boundaries) and their management. At the small scale, the REA could provide the characterisation of habitats in common topographic features (e.g. ridges and troughs), and the relationships of the communities there.

The REA must define the spatial and temporal scales at which environmental management should occur and at which natural variations occur for all environmental parameters. We currently have a very poor understanding of temporal and spatial scales for sampling to detect patterns or change in the deep sea. A nested approach may be required to define each. The REA should contain a risk assessment of which environmental variables might change, and assign sampling to contractors (and the regulator) to assess and monitor these changes. A temporal plan for the periodic review and updating of the REA must be set in order to maintain its value, and attendees suggested that this process should occur with any major change (e.g. submission of environmental data of any kind from a contractor, collection of additional environmental data, major changes to mining technology, additional scientific studies, contributions from other industries/stakeholders, etc.), or at 5 year intervals in the absence of such a change.

The REA must include all contractors' environmental data at any given time, and a systematic review and knowledge gap analysis should be used to define areas and timescales over which more information is required to complete the regional-scale 
understanding of the environment. Targets for the collection of this additional data should be set, and include both information from contractors and any necessary additional sampling or data collection. The REA should include data collected with the latest technology and methods. Attendees also suggested that the REA include a list of regional stakeholders to be consulted.

Two other ideas for the usage of the REA were suggested. The first was to increase preserved/conservation areas by coordinating the locations of PRZs along the boundaries of adjacent contractor claim areas. This may be particularly attractive when conserving areas of minable nodules or nodule densities. Caution was advised regarding joining PRZs in case this reduces the overall area preserved. The second idea was to use the regional assessment of resource coverage/density to consider relationships between ecological communities and resource presence, and to potentially set thresholds relating to resource abundance/density for mining within a claim, allowing additional areas to be preserved.

Attendees identified a number of concerns, including the time, resources and cost associated with developing a robust REA. Currently, EIA is being done in the absence of an REA, so the REA is needed urgently. Again, attendees pointed out that the success of environmental management of DSM, through REA, is dependent on unrestricted access to all environmental data collected by contractors. Concern was raised over who would pay for joint data collection and meta-analysis. Groups of contractors have done so in other industries (e.g. UK Marine Aggregate Consortium), with the particular incentive of increasing the likelihood of EIA acceptance.

These questions were used to focus the discussion on APEls:

- What information should be included in an assessment of the APEI? At what spatial and temporal scales should the data be collected? How could this data be collected (and by whom)?

- How would the APEI be used to inform the REA? Would this require changes to content or timeline for data collection or updating the assessment?

- How would the assessment of the APEI be used to evaluate contractors' baseline studies, EIA and EMPs? Would this require changes to content or timeline for data collection or updating the assessment?

- How might the assessment of APEls be different for other types of mining?

- How often should the assessment of the APEI be updated?

- Should the development of the assessment of the APEI coincide with the development of EIAs for claim areas within the same region? If so, how/in which parts?

- If the APEls in the CCZ were moved in the future or new APEls developed in other global locations, should the content or timing of data collection/assessment for these new locations be different than for the existing nodule mining-related APEIs? If so, how? 
Attendees differed on their interpretation of aims of the APEIs, and agreed that their scope, development and maintenance should be reviewed. Environmental data from the APEIs could be used to identify regional change and large scale variation as part of the REA. It could also be used for comparison with the baselines of the claim areas, and to assess impacts on the claims. In addition to their role for conservation, the APEls could be used for scientific research. Some attendees questioned the necessity of the APEIs, without a specified use and purpose.

Regardless of their additional purposes, some level of assessment and monitoring of the APEls will be required to determine their suitability and ensure their conservation status. Very little environmental data is available on the APEls, but preliminary data from APEI-6 suggest the habitats and communities there may not be similar to those in claim areas. Thus, it was agreed that the APEls must be assessed to determine whether they can be considered representative of the claim areas, or whether changes or additions to the APEIs are required to make them representative. All habitats within claim areas should be found in the APEls. This can only be determined by assessing the APEls and the claim areas sufficiently. APEls could also be adjusted or reallocated using more environmental data, including community metrics or connectivity, rather than geographic parameters. Ideally, the location and size of APEls should be set and made permanent before claims are allocated, but certainly before exploitation begins in the CCZ, to ensure their protection from mining impacts.

The APEls may be the only areas where regular planned sampling takes place in the CCZ, particularly if mining occurs sporadically. APEls present the opportunity to collect regional scale data that can be synthesised with other data to inform the REA. Data collection within the APEI should therefore answer all the objectives of the REA, and could be used to further define conservation objectives of the REA. APEls will also be useful for direct comparison at a claim scale, and the relationship between preservation reference zones within the claim zones and the APEls should be considered. In the long term, data from the APEls may be useful as a comparison when considering cumulative or combined effects from mining at the REA scale, and for characterising long-term trends in natural processes.

The regulator must manage the strategic and practical aspects of the assessment of the APEls. The iterative assessment (and monitoring) of the APEls should follow a nested approach, both spatially and temporally, and be replicable. It should include areas that could be compared to defined areas of mining impact (i.e. areas of similar habitat to those selected for mining, and similar habitats to those selected as reference areas within claims), and assessment in a similar rigorous manner to that of claims (e.g. methods, frequency), to facilitate comparison. However, in order to be comparable to all mining claims (and ensure their conservation), the assessment and monitoring of the APEls would need to continue for the duration of all mining activities at all claims. Further assessment of the APEI should be done to satisfy the aims of the REA. Other data collection at the APEIs could be used for 'blue sky science' questions, to reduce ignorance of other unknown or undefined processes. 
Ideally, qualified independent scientists would carry out the assessment of the APEIs. However, this may be difficult to implement. Several mechanisms were suggested to facilitate data collection at the APEls, including assigning some APEI sampling to contractors, or having states/contractors pay into a fund to support an independent party to collect the data.

\section{Overall opinions of environmental management for deep-sea mining}

Following the discussion sessions, attendees were surveyed about their overall opinions of environmental management. Attendees indicated that they would prioritise (in order) the improvement of REA requirements (42\%), EMP requirements $(25 \%)$, the definition of areas $(17 \%)$, and EIA requirements $(17 \%, n=12)$. In terms of areas to which science could contribute, attendees prioritise the EIA and EMP (36\%), and the REA (23\%), while $38 \%$ indicated improvements to all phases $(n=13)$. In prioritising improvements to scientific knowledge for environmental data management $(n=13)$, attendees favoured spatial assessment at the regional scale (38\%) and at the claim scale $(31 \%)$, over spatial assessment of APEls (8\%) and temporal assessment at the regional scale (8\%) or claim scale $(0 \%)$. Two attendees (16\%) voted for 'other', and stated that any further information would be helpful. Finally, attendees were surveyed as to their greatest concern for environmental management, which science could play a role in addressing. The greatest concern was for insufficient baseline knowledge prior to the start of extractive activities $(46 \%)$, procurement of competent people to evaluate environmental data / EIAs / EMPs (23\%), the development of science- and evidence-based guidance (15\%), and the collection of robust and standardised environmental data $(15 \%, n=13)$.

\section{Changes to impressions over the workshop}

Attendees' impressions of environmental management of DSM fluctuated over the workshop (Fig. 1). However, there was general agreement that the environmental management structure is not 'completely' ready for DSM (zero votes, Fig. 1a), and that the MIDAS project will guide the policy to some degree (zero votes for 'slightly' and 'not at all' in Fig. 1d). Confidence that policy is being guided by science was moderate (no votes for 'completely' or 'not at all' after the workshop start, Fig. 1c). Interestingly, the only area where a negative trend was detected (that is, where the slope of a fitted linear regression was significantly negative, $-0.172, R^{2}=0.859, n=4$ ), was the attendees' confidence in MIDAS influencing environmental management policy for DSM. In discussion, it was identified that many attendees became aware of gaps in management policy over the course of the workshop, which may complicate this result. 

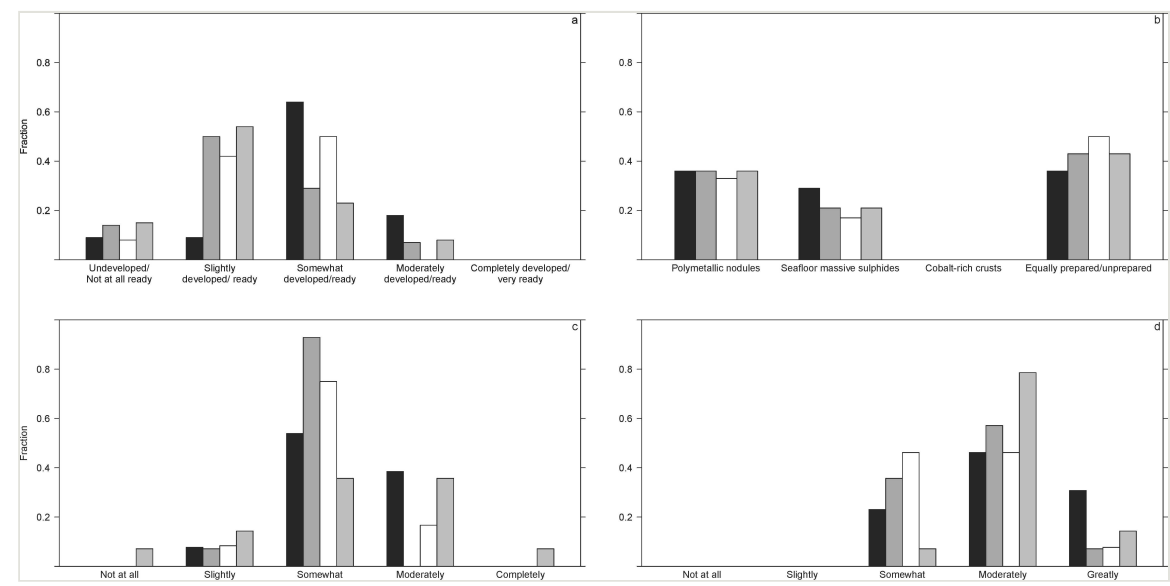

Figure 1.

Histograms of responses from workshop attendees at four time points over the workshop, Start of workshop (black), End of Day 1 (dark grey), End of Day 2 (white), End of Day 3 (light grey): (a) How developed is the environmental management of deep-sea mining? (b) Which type of deep-sea mining is best prepared in terms of environmental management? (c) To what extent do you think environmental management policy for deep-sea mining is being guided by science? (d) To what extent do you think the MIDAS project is/will guide environmental management policy?

\section{Conclusions}

The use of the scenario as a basis for the evaluation of protocols was unique amongst workshops on the environmental management of DSM. Feedback from attendees suggested that it focused discussions, allowed risks to be evaluated, exposed true knowledge gaps, and reduced uncertainties and generalisations. Thus, reasoned evaluations were achieved.

A common thread in the workshop discussions was the need for sufficient resources to undertake environmental management and assessment. Resources (staff, time and money) are needed at the regulatory level to establish the protocols required, maintain the REAs and APEls, regularly engage the states/contractors, receive and review data and analyses provided to the states/contractors, perform analyses, provide sound decisions, and audit contractors activities. Contractors must provide sufficient resources to manage and assess the environment at the claim level, including data collection and interpretation, report preparation, management design and implementation, for maintenance of a robust conceptual model including baseline characterisation, EIA preparation and operational monitoring. This presents a difficulty in the procurement of competent personnel to carry out this work. In other industries, independent environmental specialists are contracted. To date, much of the environmental assessment work for DSM has been completed by academic researchers. However, long-term repeat monitoring may not continue to be 
attractive from an academic point of view. This could be an opportunity for capacity building and knowledge transfer, if the regulator plans for involvement of trainees from member states.

Another common concern was the implementation of appropriate legal and financial mechanisms to facilitate this process, including allowances for regular review and revision, and adaptive management. Legal experts should be consulted on the details of this point, but workshop attendees were keen to point out that this is critical to successful environmental management of DSM.

\section{Grant title}

This work forms part of the Managing Impacts of Deep-seA reSource exploitation (MIDAS) project funded by the European Union Seventh Framework Programme (FP7/2007-2013) grant agreement $n^{\circ} 603418$.

\section{References}

- International Seabed Authority (2011) Environmental Management Needs for Exploration and Exploitation of Deep Sea Minerals, ISA Technical Study: No. 10. International Seabed Authority, Nadu, Fiji, 52 pp.

- Lodge M, Johnson D, Gurun GL, Wengler M, Weaver P, Gunn V (2014) Seabed mining: International Seabed Authority environmental management plan for the ClarionClipperton Zone. A partnership approach. Marine Policy 49: 66-72. DOI: 10.1016/ j.marpol.2014.04.006

- $\quad$ Oebius HU, Becker HJ, Rolinski S, Jankowski JA (2001) Parametrization and evaluation of marine environmental impacts produced by deep-sea manganese nodule mining. Deep Sea Research Part II: Topical Studies in Oceanography 48: 3453-3467. DOI: 10.1 016/S0967-0645(01)00052-2

- $\quad$ Smith CR, Levin LA, Koslow A, Tyler PA, Glover AG (2008) The near future of the deep seafloor ecosystems. In: Polunin N (Ed.) Aquatic Ecosystems: Trends and Global Prospects. Cambridge University Press, Cambridge.

- Underwood AJ (1992) Beyond BACl: the detection of environmental impacts on populations in the real, but variable, world. Journal of Experimental Marine Biology and Ecology 161 (2): 145-178. DOI: 10.1016/0022-0981(92)90094-Q

- Wedding LM, Friedlander AM, Kittinger JN, Watling L, Gaines SD, Bennett M, Hardy SM, Smith CR (2013) From principles to practice: a spatial approach to systematic conservation planning in the deep sea. Proceedings of the Royal Society of London B: Biological Sciences 280 (1773): 20131684. DOI: 10.1098/rspb.2013.1684 